\title{
Recent Advances in Symmetry Analysis and Exact Solutions in Nonlinear Mathematical Physics
}

\author{
Maria Bruzón, ${ }^{1}$ Chaudry M. Khalique, ${ }^{2}$ Maria L. Gandarias, \\ Rita Traciná, ${ }^{3}$ and Mariano Torrisi ${ }^{3}$ \\ ${ }^{1}$ University of Cádiz, Cádiz, Spain \\ ${ }^{2}$ North-West University, Vanderbijlpark, South Africa \\ ${ }^{3}$ Università di Catania, Catania, Italy \\ Correspondence should be addressed to Maria Bruzón; m.bruzon@uca.es
}

Received 4 April 2017; Accepted 5 April 2017; Published 16 April 2017

Copyright (C) 2017 Maria Bruzón et al. This is an open access article distributed under the Creative Commons Attribution License, which permits unrestricted use, distribution, and reproduction in any medium, provided the original work is properly cited.

Partial differential equations are used to describe a wide variety of physical phenomena such as fluid dynamics, plasma physics, solid mechanics, and quantum field theory that arise in physics. Many of these equations are nonlinear and, in general, these equations are often very difficult to solve explicitly. Many systematic methods are usually employed to study the nonlinear equations: these include the generalized symmetry method, the Painlevé analysis, the inverse scattering method, the Bäcklund transformation method, the conservation law method, the Cole-Hopf transformation, and the Hirota bilinear method.

Constructing exact solutions, in particular travelling wave solutions, of nonlinear equations plays an important role in soliton theory. Several important direct methods have been developed for obtaining travelling wave solutions to nonlinear partial differential equations such as the inverse scattering method, the tanh-function method, the extended tanh-function method, the $G^{\prime} / G$ method, the simplest method, and the modified simplest method.

The authors of this special issue had been invited to submit original research articles as well as review articles in the following topics: methods for obtaining solutions of partial differential equations; conservation laws; general methods for obtaining solutions of ordinary differential equations; travelling wave solutions; novel applications in physics.

However, we received 22 papers in these research fields. After a rigorous reviewing process, six articles were finally accepted for publication. These articles contain some new and innovative techniques and ideas that may stimulate further researches in several branches of theory and applications of the transformation groups.

In "The Rational Solutions and Quasi-Periodic Wave Solutions as well as Interactions of $\mathrm{N}$-Soliton Solutions for $3+$ 1 Dimensional Jimbo-Miwa Equation," H. Yang et al. analyze the rational solutions, quasi-periodic wave solutions obtained by the Hirota method and the theta function for the $3+1$ dimensional Jimbo-Miwa equation. The knowledge of these solutions allows them to explain the interaction of the $N$ soliton solutions, that is, to show when the soliton solution can be changed into the resonant solution and when there will appear the pursue collision; that is, the soliton with the faster speed will catch up with the soliton with the slower speed (after the collision, the two solitons will continue to spread as the previous speed and the direction).

In "An Efficient Numerical Method for the Solution of the Schrödinger Equation," L. Zhang and T. E. Simos show the original development of a new five-stage symmetric two-step fourteenth-algebraic-order method with vanished phase-lag and its first, second, and third derivatives. More specifically, they firstly introduce the development of the new method and the determination of the local truncation error and successively the local truncation error analysis and the stability. Finally, the interval of periodicity analysis and the efficiency of the new method are considered by applying it to couple of Schrödinger equations. 
In the paper titled "Asymptotic Expansion of the Solutions to Time-Space Fractional Kuramoto-Sivashinsky Equations," the attention of authors is devoted to finding the asymptotic expansion of solutions to fractional partial differential equations with initial conditions. The residual power series method is proposed for time-space fractional partial differential equations, where the fractional integral and derivative are described in the sense of Riemann-Liouville integral and Caputo derivative. They apply the method to the linear and nonlinear time-space fractional Kuramoto-Sivashinsky equation with initial value and obtain asymptotic expansion of the solutions, which confirm the accuracy and efficiency of the method.

In "New Periodic Solutions for a Class of Zakharov Equations," C. Sun and S. Ji consider a class of nonlinear Zakharov equations. Inspired by Angluo's idea, by applying the Jacobian elliptic function method, they obtain new periodic solutions for Klein-Gordon Zakharov equations, Zakharov equations, and Zakharov-Rubenchik equations.

In the paper titled "Stability of the Cauchy Additive Functional Equation on Tangle Space and Applications," S. $\mathrm{H}$. Kim introduces real tangles (a generalization of rational tangles) and its operations to enumerating tangles by using the calculus of continued fraction. Moreover, he studies about the analytical structure of tangles, knots, and links by using new operations between real tangles which need not have the topological structure. As for applications of the analytical structure, he proves the generalized Hyers-Ulam stability of the Cauchy additive functional equation $f(x \oplus y)=f(x) \oplus$ $f(y)$ in tangle space which is a set of real tangles with analytic structure and describes the DNA recombination as the action of some enzymes on tangle space.

In their paper titled "The Stochastic Resonance Behaviors of a Generalized Harmonic Oscillator Subject to Multiplicative and Periodically Modulated Noises," S. Zhong et al. study the stochastic resonance (SR) characteristics of a generalized Langevin linear system driven by a multiplicative noise and a periodically modulated noise. They take in consideration a generalized Langevin equation (GLE) driven by an internal noise with long-memory and long-range dependence, such as fractional Gaussian noise and MittagLeffler noise. Such a model is appropriate to characterizing the chemical and biological solutions as well as to some nanotechnological devices. An exact analytic expression of the output amplitude is obtained. Based on it, some characteristic features of stochastic resonance phenomenon are revealed. On the other hand, by the use of the exact expression, they obtain the phase diagram for the resonant behaviors of the output amplitude versus noise intensity under different values of system parameters. These results could give the theoretical basis for practical use and control of the SR phenomenon of this mathematical model in future works.

\section{Acknowledgments}

We would like to thank all the authors who sent their works and all the referees for the time spent in reviewing the papers. Their contributions and their efforts have been very important for the publication of this special issue.

Maria Bruzón Chaudry M. Khalique Maria L. Gandarias Rita Traciná Mariano Torrisi 


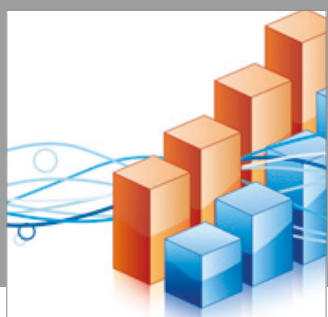

Advances in

Operations Research

vatersals

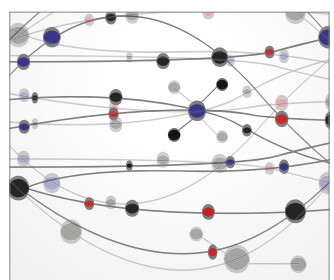

\section{The Scientific} World Journal
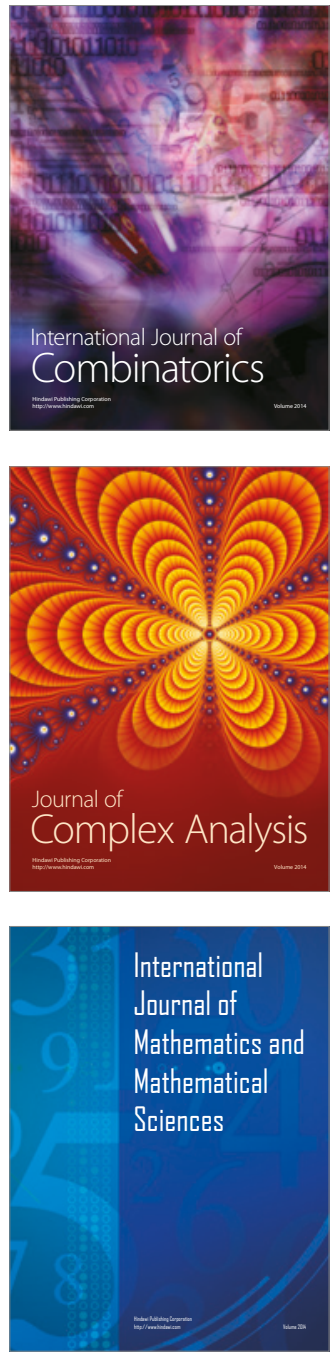
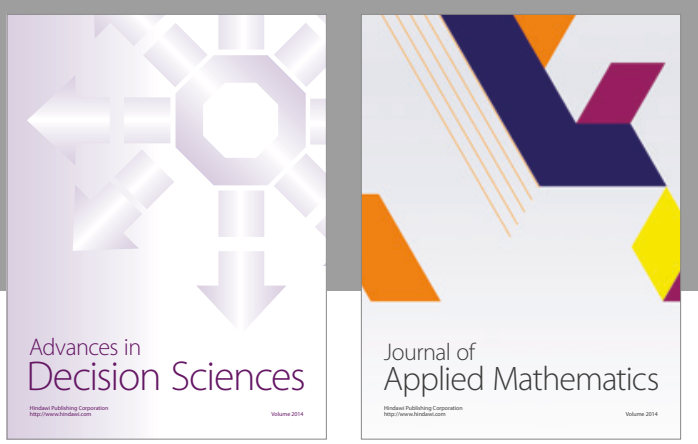

Algebra

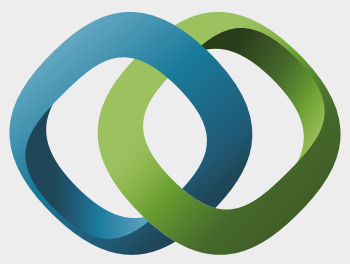

\section{Hindawi}

Submit your manuscripts at

https://www.hindawi.com
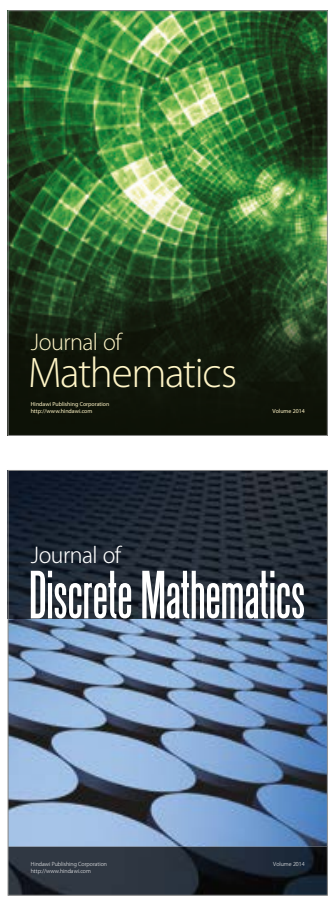

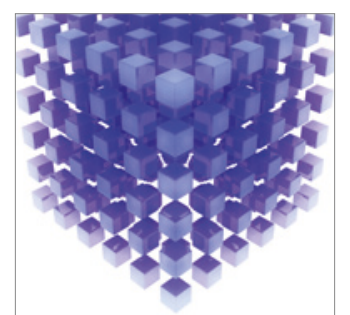

Mathematical Problems in Engineering
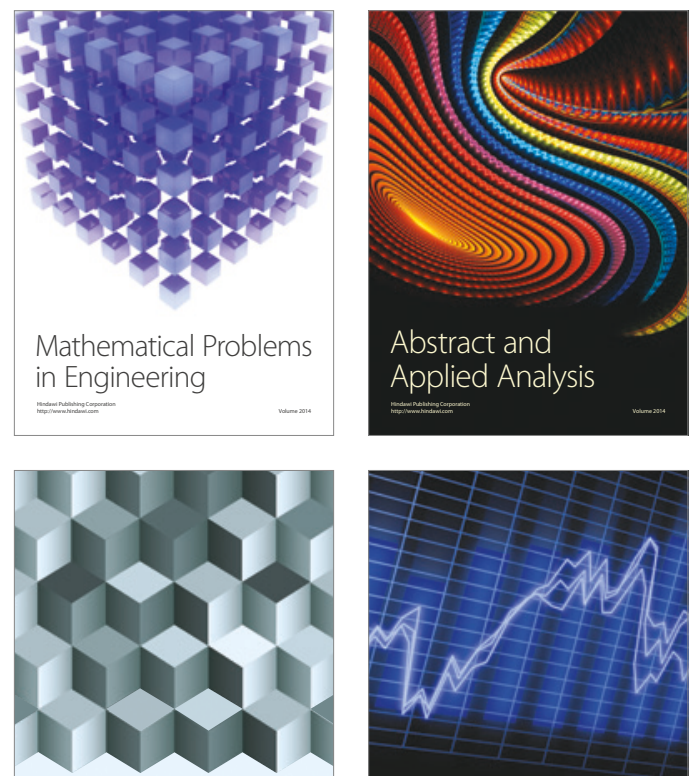

Journal of

Function Spaces

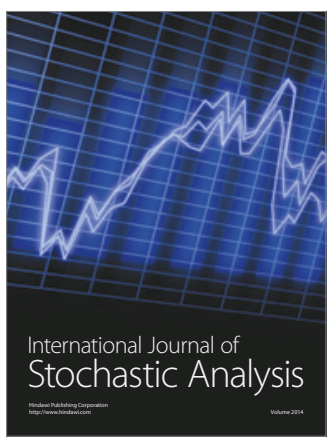

Probability and Statistics
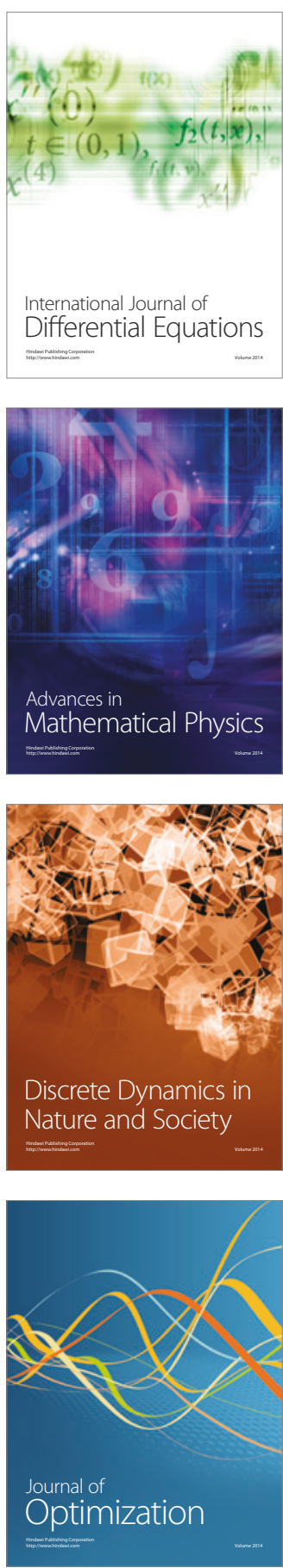